\title{
A Unified Approach for Processing Unbalanced Conditions in Transient Stability Calculations
}

\author{
Xuefeng Bai, Tong Jiang, Zhizhong Guo, Zheng Yan, and Yixin Ni, Senior Member, IEEE
}

\begin{abstract}
A unified approach has been proposed in the paper for processing unbalanced operating conditions in transient stability calculations. The suggested method combined advantages of both abc phase coordinates and 012 sequence coordinates. The former is used to describe faults since it is suitable to modeling simultaneous faults and the latter is used for time simulation for its sparse nature. Novel fault description and coordinate conversion formulation is presented. The equivalent positive sequence bus admittance matrix is derived for interface with generators in stability simulation. With the new method, the sequence network constitution can be avoided; multiple and simultaneous faults and element parameter unbalance can be simulated easily; and the sparse technology can still be applied for study efficiency. Computer test results show clearly the effectiveness and efficiency of the new method.
\end{abstract}

Index Terms-Phase components, short-circuit analysis, symmetrical components, transient stability calculation.

\section{INTRODUCTION}

$\mathbf{I}$ $\mathrm{N}$ power systems, three-phase unbalanced operations may occur due to asymmetrical system parameters and asymmetrical operating conditions. Unbalanced system parameters may exist in system apparatus such as transmission lines without perfect conductor commutation; while unbalanced operating conditions are mainly caused by asymmetrical shunt (short circuit) or series (open conductor) faults.

The symmetrical components method (SCM) is widely used for asymmetric faults analysis and transient stability study under asymmetric disturbances. The method is based on the principle that under a three-phase unbalanced fault, the faulted threephase network can be expressed by three decoupled but properly connected sequence networks [1]. But it should be stressed that the conversion from an abc phase-component network to the corresponding decoupled 012 sequence-component network is only applicable in systems with symmetrical element parameters. For systems with asymmetrical element parameters, the sequence-networks cannot be decoupled completely and the SCM cannot be used in such systems. Otherwise the asymmetry of element parameters has to be ignored which might introduce noticeable errors when element asymmetry is severe. This problem

Manuscript received May 17, 2004; revised April 3, 2005. This work was supported in part by the National Natural Science Foundation of China under Project 50307003 and in part by the National Key Basic Research Special Fund under Project 2004CB217900. Paper no. TPWRS-00263-2004.

X. Bai, T. Jiang, and Z. Guo are with the Department of Electrical Engineering, Harbin Institute of Technology, Harbin 150001, China (e-mail: xfbai@eee.hku.hk)

Z. Yan is with the Department of Electrical Engineering, Shanghai Jiaotong University, Shanghai 200030, China (e-mail: zyan@eee.hku.hk)

$\mathrm{Y}$. Ni is with the Department of Electrical and Electronic Engineering, University of Hong Kong, Hong Kong, China (e-mail: yxni@eee.hku.hk)

Digital Object Identifier 10.1109/TPWRS.2005.857922 greatly affects the application of sequence component method in power system fault analysis and transient stability simulation. Moreover, the SCM handles shunt (or series) unbalances and faults by building up one-port (or two-port) Thevenin equivalents in both fault analysis and transient stability calculations [2], [3]. However the formation of Thevenin equivalents for simultaneous-faults are cumbersome and difficult [4]. Therefore the author of [5] said "strictly speaking, the traditional sequence component method is no longer applicable in most complicated areas in power engineering."

On the other hand, the phase components method (PCM) deals with the faults in accordance with the boundary conditions of the three phases. It is convenient to consider all possible faults with clear physical concepts, encompassing simultaneous symmetrical and asymmetrical short circuit and/or open conductor faults [6], [7]. In [8], fault models, and relevant algorithms were summarized in matrix form, by which a unified mathematical formulation and a standard solution method for any complex multifault calculations are formed. However, the coupling of abc phase components greatly sacrifices the efficiency of fault analysis and transient stability simulation in phase coordinates.

Apparently, it is of particular significance to combine the advantages of both SCM and PCM so that the resultant new method can handle asymmetrical network and complex network manipulations flexibly and efficiently in fault analysis and transient stability simulation. This issue is addressed in the paper.

In this paper, a unified approach is proposed for the purpose. It uses abc coordinates in asymmetric element and fault descriptions without establishing sequence networks. The abc coordinates are then properly converted to 012 coordinates for efficient time simulation. The equivalent positive sequence bus admittance matrix for interface to the generators and dynamic loads in time simulation is derived from the bus admittance matrix in 012 coordinates through Gauss-Jordan elimination method. Computer test results show that the suggested unified approach works efficiently with good accuracy.

\section{SHORT-CIRCUIT FAULT DESCRIPTION}

As we all know, most of the elements in power systems have symmetric parameters, and under asymmetrical faults sequence component model can reduce calculation significantly as compared with phase component model. Therefore in the paper the sequence models are adopted for symmetrical elements including generators and symmetric loads, transformers and transmission lines [9], [10]. For asymmetrical network elements, e.g., some noncommutated lines, the phase component models are adopted with the coupling of three phases expressed directly [11]. 
For simplicity, we first present the proposed model for power networks with symmetric three phase elements. The power networks with asymmetrical elements will be discussed later. For a symmetrical three-phase power network, the network equation in abc phase coordinates takes the form (all in per unit $(\mathrm{pu})$ and complex numbers):

$$
Y^{(a b c)} V^{(a b c)}=I^{(a b c)} .
$$

That is

$$
\left[\begin{array}{cccc}
Y_{11} & Y_{12} & \cdots & Y_{1 n} \\
Y_{21} & Y_{22} & \cdots & Y_{2 n} \\
\cdots & \cdots & \cdots & \cdots \\
Y_{n 1} & Y_{n 2} & \cdots & Y_{n n}
\end{array}\right]\left[\begin{array}{c}
V_{1} \\
V_{2} \\
\cdots \\
V_{n}
\end{array}\right]=\left[\begin{array}{c}
I_{1} \\
I_{2} \\
\cdots \\
I_{n}
\end{array}\right]
$$

where

$$
\begin{aligned}
Y_{i j} & =\left[\begin{array}{lll}
y_{i j}^{(a a)} & y_{i j}^{(a b)} & y_{i j}^{(a c)} \\
y_{i j}^{(b a)} & y_{i j}^{(b b)} & y_{i j}^{(b c)} \\
y_{i j}^{(c a)} & y_{i j}^{(c b)} & y_{i j}^{(c c)}
\end{array}\right] \\
V_{j} & =\left[\begin{array}{l}
V_{j}^{(a)} \\
V_{j}^{(b)} \\
V_{j}^{(c)}
\end{array}\right] \quad I_{j}=\left[\begin{array}{c}
I_{j}^{(a)} \\
I_{j}^{(b)} \\
I_{j}^{(c)}
\end{array}\right], \quad(i, j=1,2, \ldots, n) .
\end{aligned}
$$

A single line-to-ground (1L-G) fault at bus $j$ is used as an example to demo the modeling of short circuit faults with phase A as the faulted phase. The fault can be regarded as a current injection at bus $j$ denoted as $I_{s c}$, which makes the faulted bus phase A voltage equal to zero. Hence, (1) can be rewritten as

$\left[\begin{array}{cccccc}Y_{11} & Y_{12} & \ldots & Y_{1 j} & \ldots & Y_{1 n} \\ Y_{21} & Y_{22} & \ldots & Y_{2 j} & \ldots & Y_{2 n} \\ \ldots & \ldots & \ldots & \ldots & \ldots & \ldots \\ Y_{j 1} & Y_{j 2} & \ldots & Y_{j j} & \ldots & Y_{j n} \\ \ldots & \ldots & \ldots & \ldots & \ldots & \ldots \\ Y_{n 1} & Y_{n 2} & \ldots & Y_{n j} & \ldots & Y_{n n}\end{array}\right]\left[\begin{array}{c}V_{1} \\ V_{2} \\ \ldots \\ V_{j} \\ \ldots \\ V_{n}\end{array}\right]=\left[\begin{array}{c}I_{1} \\ I_{2} \\ \ldots \\ I_{j} \\ \ldots \\ I_{n}\end{array}\right]+\left[\begin{array}{c}0 \\ 0 \\ \ldots \\ I_{s c} \\ \ldots \\ 0\end{array}\right]$

where

$$
I_{s c}=\left[\begin{array}{lll}
I_{f}^{(a)} & 0 & 0
\end{array}\right]^{T} \text { and } V j=\left(\begin{array}{lll}
0 & V_{j}^{(b)} & V_{j}^{(c)}
\end{array}\right)^{T} .
$$

By moving $I_{s c}$ to the left-hand side of (3), we obtain

$$
\left[\begin{array}{cccccc}
Y_{11} & Y_{12} & \ldots & Y_{1 j}^{\prime} & \ldots & Y_{1 n} \\
Y_{21} & Y_{22} & \ldots & Y_{2 j}^{\prime} & \ldots & Y_{2 n} \\
\ldots & \ldots & \ldots & \ldots & \ldots & \ldots \\
Y_{j 1} & Y_{j 2} & \ldots & Y_{j j}^{\prime} & \ldots & Y_{j n} \\
\ldots & \ldots & \ldots & \ldots & \ldots & \ldots \\
Y_{n 1} & Y_{n 2} & \ldots & Y_{n j}^{\prime} & \ldots & Y_{n n}
\end{array}\right]\left[\begin{array}{c}
V_{1} \\
V_{2} \\
\ldots \\
V_{j}^{\prime} \\
\ldots \\
V_{n}
\end{array}\right]=\left[\begin{array}{c}
I_{1} \\
I_{2} \\
\ldots \\
I_{j} \\
\ldots \\
I_{n}
\end{array}\right]
$$

where the equivalent fault bus voltage vector $V_{j}^{\prime}$ is defined as $V_{j}^{\prime}=\left[\begin{array}{lll}I_{f}^{(a)} & V_{j}^{(b)} & V_{j}^{(c)}\end{array}\right]^{T}$; and the $j$ th block-column elements of $Y$-matrix should be

$$
\begin{aligned}
Y_{i j}^{\prime} & =\left[\begin{array}{lll}
0 & y_{i j}^{(a b)} & y_{i j}^{(a c)} \\
0 & y_{i j}^{(b b)} & y_{i j}^{(b c)} \\
0 & y_{i j}^{(c b)} & y_{i j}^{(c c)}
\end{array}\right] \quad(i \neq j) \\
Y_{j j}^{\prime} & =\left[\begin{array}{ccc}
-1 & y_{j j}^{(a b)} & y_{j j}^{(a c)} \\
0 & y_{j j}^{(b b)} & y_{j j}^{(b c)} \\
0 & y_{j j}^{(c b)} & y_{j j}^{(c c)}
\end{array}\right] .
\end{aligned}
$$

Comparing (4) with (2), it can be found that

$$
\left\{\begin{array}{l}
Y_{i j}^{\prime}=Y_{i j}\left[\begin{array}{lll}
0 & 0 & 0 \\
0 & 1 & 0 \\
0 & 0 & 1
\end{array}\right] \triangleq Y_{i j} T_{1} \quad(i \neq j) \\
Y_{j j}^{\prime}=Y_{j j}\left[\begin{array}{lll}
0 & 0 & 0 \\
0 & 1 & 0 \\
0 & 0 & 1
\end{array}\right]+\left[\begin{array}{ccc}
-1 & 0 & 0 \\
0 & 0 & 0 \\
0 & 0 & 0
\end{array}\right] \triangleq Y_{j j} T_{1}+T_{2}
\end{array}\right.
$$

where $T_{1}$ and $T_{2}$ are named as the conversion matrices of the $1 \mathrm{~L}-\mathrm{G}$ fault.

The conversion matrices and equivalent fault bus voltage vectors for various short-circuit faults are given in Table I with phase A as the reference phase. If the reference phase is not phase A, the relevant expressions can be derived similarly without difficulty.

The above step shows how to express a short-circuit fault using abc phase components in the suggested approach. It is clear that there is no need to form sequence networks and connect them properly according to the fault type. Also, the new approach is convenient to describe simultaneous faults in the systems. However, we know from power system dynamics that the generator rotor dynamics are mainly related to network positive sequence components, therefore in transient stability simulation we have to provide equivalent positive sequence network equation to interface to generator models. Besides the time simulation of system transients in abc phase coordinates is inefficient for the coupling of three phase circuits. Therefore, we need to convert (4) and (5) from abc phase coordinates to 012-sequence coordinates to make efficient time simulation and then derive equivalent positive sequence network equations for generator interface, which will be studied in the next section.

\section{Network SOlution AlgORITHM}

\section{A. Conversion of the Network Equation From abc Phase Coordinates to 012 Sequence Coordinates}

For (4) if the variation of bus admittance matrix caused by asymmetrical faults is $\Delta Y^{(a b c)}$, the network equation under fault-on condition can be written as

$$
\left(Y^{(a b c)}+\Delta Y^{(a b c)}\right) V^{(a b c)}=I^{(a b c)}
$$

where $\Delta Y^{(a b c)}$ has only nonzero elements in jth block-column when bus $j$ is the fault bus with the nonzero elements calculated by [see (5)]

$$
\begin{aligned}
& \Delta Y_{i j}^{(a b c)}=-Y_{i j}^{(a b c)}+Y_{i j}^{(a b c)} T_{1} \quad(i \neq j) \\
& \Delta Y_{j j}^{(a b c)}=-Y_{j j}^{(a b c)}+Y_{j j}^{(a b c)} T_{1}+T_{2} .
\end{aligned}
$$

Next, based on abc-012 coordinates conversion matrix

$$
T=\frac{1}{3}\left[\begin{array}{ccc}
1 & 1 & 1 \\
1 & \alpha & \alpha^{2} \\
1 & \alpha^{2} & \alpha
\end{array}\right] \quad\left(\alpha=e^{j 120^{\circ}}\right)
$$


Equation (6) can be converted from abc phase coordinates to 012 sequence coordinates through the following transformations:

$$
\begin{aligned}
& V^{(012)}=T V^{(a b c)} \quad I^{(012)}=T I^{(a b c)} \\
& Y^{(012)}=T Y^{(a b c)} T^{-1} \\
& \text { and } \quad T_{1}^{(012)}=T T_{1} T^{-1} \quad T_{2}^{(012)}=T T_{2} T^{-1} \text {. }
\end{aligned}
$$

Hence, (6) will be converted to 012 coordinates and denoted as

$$
\left(Y^{(012)}+\Delta Y^{(012)}\right) V^{(012)}=I^{(012)}
$$

where

$$
\begin{aligned}
& \Delta Y_{i j}^{(012)}=-Y_{i j}^{(012)}+Y_{i j}^{(012)} T_{1}^{(012)} \quad(i \neq j) \\
& \Delta Y_{j j}^{(012)}=-Y_{j j}^{(012)}+Y_{j j}^{(012)} T_{1}^{(012)}+T_{2}^{(012)} .
\end{aligned}
$$

It is clear that in the bus admittance matrix demoted by $\left(Y^{(012)}+\Delta Y^{(012)}\right)$ only the 3 by 3 submatrices in the $j$ th block-column (if bus $\mathrm{j}$ is the faulted bus) are asymmetrical and it cannot be decoupled in sequence coordinates through abc-012 coordinates conversion. By exchanging the $j$ th block-column with the $n$th block-column and the $j$ th block-row with the $n$th block-row respectively (the corresponding voltage and current variables in $V^{(012)}$ and $I^{(012)}$ will be exchanged accordingly as well), (7) will be

$$
\left[\begin{array}{cc}
Y_{S S} & Y_{S U} \\
Y_{U S} & Y_{U U}
\end{array}\right]\left[\begin{array}{c}
V_{S} \\
V_{U}
\end{array}\right]=\left[\begin{array}{c}
I_{S} \\
I_{U}
\end{array}\right]
$$

where $Y_{S S}$ and $Y_{U S}$ are relevant to symmetrical element parameters only. It is easy to prove their $3 \times 3$ block-entries are diagonal matrices with the form:

$$
Y_{i j}=\left[\begin{array}{lll}
y_{i j}^{(0)} & & \\
& y_{i j}^{(1)} & \\
& & y_{i j}^{(2)}
\end{array}\right] \quad(j \in S)
$$

while $Y_{S U}$ and $Y_{U U}$ in (7) are relevant to fault bus $j$. The equivalent faulted bus voltage $V_{U}$ in abc coordinates is shown in Table I. Therefore the 3 by 3 block entries of $Y_{S U}$ and $Y_{U U}$ are affected by the unbalanced operating conditions and cannot be decoupled in 012 coordinates, i.e.,

$$
Y_{i j}=\left[\begin{array}{lll}
y_{i j}^{(00)} & y_{i j}^{(01)} & y_{i j}^{(02)} \\
y_{i j}^{(10)} & y_{i j}^{(11)} & y_{i j}^{(12)} \\
y_{i j}^{(20)} & y_{i j}^{(21)} & y_{i j}^{(22)}
\end{array}\right] \quad(j \in U)
$$

\section{B. Formation of Equivalent Positive Sequence Bus Admittance Matrix}

As we mentioned above, for transient stability simulation, the network equivalent positive sequence bus admittance matrix
TABLE I

CONVERSION MATRICES AND EQUIVALENT FAULT Bus Voltage Phasors For FaUlt at Bus $j$ With ReFERring Phase A

\begin{tabular}{c|c|c|c|c}
\hline $\begin{array}{c}\text { Short circuit } \\
\text { type }\end{array}$ & $T_{1}$ & $T_{2}$ & $V_{j}^{\prime}$ \\
\hline $\begin{array}{c}\text { Single line to } \\
\text { ground } \\
\text { (1L-G) }\end{array}$ & {$\left[\begin{array}{lll}0 & 0 & 0 \\
0 & 1 & 0 \\
0 & 0 & 1\end{array}\right]$} & {$\left[\begin{array}{ccc}-1 & 0 & 0 \\
0 & 0 & 0 \\
0 & 0 & 0\end{array}\right]$} & {$\left[\begin{array}{c}I_{f}^{(a)} \\
V_{j}^{(b)} \\
V_{j}^{(c)}\end{array}\right]$} \\
\hline $\begin{array}{c}\text { Double lines } \\
\text { to ground } \\
\text { (2L-G) }\end{array}$ & {$\left[\begin{array}{lll}1 & 0 & 0 \\
0 & 0 & 0 \\
0 & 0 & 0\end{array}\right]$} & {$\left[\begin{array}{ccc}0 & 0 & 0 \\
0 & -1 & 0 \\
0 & 0 & -1\end{array}\right]$} & {$\left[\begin{array}{c}V_{j}^{(a)} \\
I_{f}^{(b)} \\
I_{f}^{(c)}\end{array}\right]$} \\
\hline $\begin{array}{c}\text { Line to line } \\
\text { (L-L) }\end{array}$ & {$\left[\begin{array}{lll}1 & 0 & 0 \\
0 & 1 & 0 \\
0 & 1 & 0\end{array}\right]$} & {$\left[\begin{array}{ccc}0 & 0 & 0 \\
0 & 0 & -1 \\
0 & 0 & 1\end{array}\right]$} & {$\left[\begin{array}{c}V_{j}^{(a)} \\
V_{j}^{(b)} \\
I_{f}^{(b)}\end{array}\right]$} \\
\hline $\begin{array}{c}\text { Three phase } \\
\text { fault }\end{array}$ & {$\left[\begin{array}{lll}1 & 0 & 0 \\
1 & 0 & 0 \\
1 & 0 & 0\end{array}\right]$} & {$\left[\begin{array}{ccc}0 & -1 & 0 \\
0 & 0 & -1 \\
0 & 1 & 1\end{array}\right]$} & {$\left[\begin{array}{c}V_{j}^{(a)} \\
I_{f}^{(a)} \\
I_{f}^{(b)}\end{array}\right]$} \\
\hline $\begin{array}{c}\text { Three phase to } \\
\text { ground }\end{array}$ & {$\left[\begin{array}{lll}0 & 0 & 0 \\
0 & 0 & 0 \\
0 & 0 & 0\end{array}\right]$} & {$\left[\begin{array}{ccc}-1 & 0 & 0 \\
0 & -1 & 0 \\
0 & 0 & -1\end{array}\right]$} & {$\left[\begin{array}{c}I_{f}^{(a)} \\
I_{f}^{(b)} \\
I_{f}^{(c)}\end{array}\right]$} \\
\hline
\end{tabular}

has to be derived to interface to generators and dynamic loads, This means in (8) zero and negative sequence components and relevant variables have to be eliminated which can be implemented through Gauss-Jordan elimination. Here sparse matrix technology can be used for efficient calculation.

In real elimination, the rows and columns of bus admittance matrix in (8) have been rearranged to be

$$
Y=\left[\begin{array}{lll}
Y_{00} & Y_{02} & Y_{01} \\
Y_{20} & Y_{22} & Y_{21} \\
Y_{10} & Y_{12} & Y_{11}
\end{array}\right]
$$

where $Y_{00}, Y_{11}$, and $Y_{22}$ are submatrices corresponding to zero, positive and negative sequence network respectively. The nonzero elements in off-diagonal sub-matrices are located only in the last column relevant to the fault bus and corresponding to the elements in $Y_{S U}$ and $Y_{U U}$ in (8).

After applying Gauss-Jordan elimination to (11), the reduced bus admittance matrix takes the form

$$
Y^{\prime}=\left[\begin{array}{ccc}
Y_{00}^{\prime} & Y_{02}^{\prime} & Y_{01}^{\prime} \\
0 & Y_{22}^{\prime} & Y_{21}^{\prime} \\
0 & 0 & Y_{11}^{\prime}
\end{array}\right]
$$

where the elements in the lower triangles of $Y_{00}^{\prime}$ and $Y_{22}^{\prime}$ are zero, and their diagonal elements are $1 . Y_{11}^{\prime}$ is the required equivalent positive sequence bus admittance matrix. The influence of unbalanced faults has been included in this matrix.

$Y_{11}^{\prime}$ will be used to perform transient stability simulations. In this paper, a high-order Taylor expansion series is used to accelerate the transient stability calculations [9], [12]. During the 
network solution, the Norton equivalent currents of generator stators are used to calculate the stator voltages, and the stator currents can then be calculated.

The overall procedure of the new method for fault analysis and transient stability simulation considering asymmetric short circuit fault is summarized below.

Step 1 The short circuit fault is described in bus-admittance matrix equations in abc phase coordinates (see section II) according to the boundary conditions and then the bus admittance matrix equations is converted to the 012 sequence coordinates.

Step 2 The bus admittance matrix in 012 coordinates is reduced to generator nodes and fault nodes through Gauss-Jordan elimination to reach the form as shown in (8)-(10).

Step 3 Gauss-Jordan elimination is applied again to obtain the equivalent positive sequence bus admittance matrix [see (12)] after proper exchanges of rows and columns of bus admittance matrix [see(11)].

Some further discussions on the proposed method are given below.

1) The proposed method can avoid constitution of sequence network under asymmetric faults. It is convenience to consider simultaneous faults. The fault reference phase can be any phases. The fault description is straightforward with clear physical concepts.

2) The method can still make use of the advantages of efficient calculation under sequence component coordinates especially when most of the system elements are symmetric. Besides, sparse technology can be applied to further improve calculation efficiency.

3) When there is an asymmetrical element in the network, the entries of this element should be placed at the end of the bus admittance matrix. The generator nodes, fault nodes and asymmetrical element nodes should be kept during the elimination. The other process is the same as that described above.

4) When there is an asymmetric open conductor fault, it can be handled similarly as an asymmetric element.

5) Comparing the new method with conventional pure symmetrical component domain analysis, the additional computation of the new method is mainly on the formation of equivalent positive sequence admittance matrix by Gauss-Jordan elimination. (In conventional method, equivalent fault impedance will be worked out and insert to the positive sequence network to simulate the zero and negative components impacts on system stability.) The computer test results presented in the next section show that the extra computation is very small and cannot be felt in the tested cases.

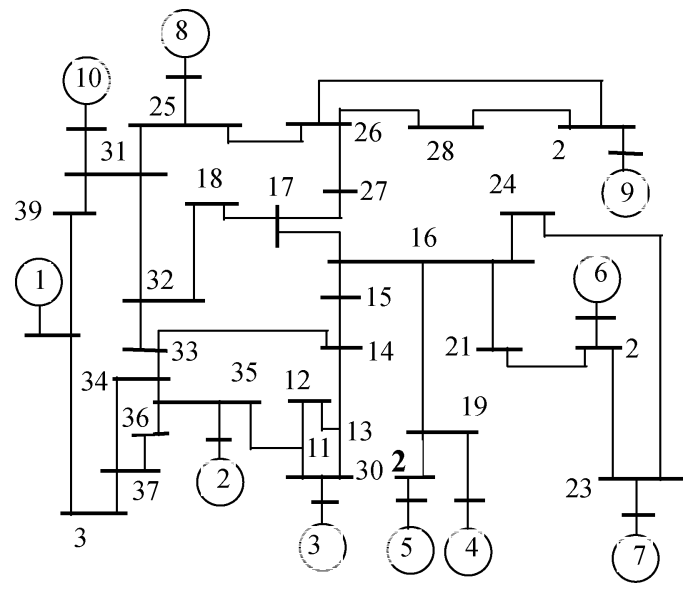

Fig. 1. Test system 1 (buses reordered).

\section{NuMERICAL TeSt Results}

\section{A. Results From Test System 1}

The IEEE New England 10-machine, 39-bus system is adopted as test system 1 . The single-line diagram of the system is shown in Fig. 1.

The fault is applied at the from-bus of line 15-16 at time $\mathrm{t}=0 \mathrm{~s}$ and cleared at time $\mathrm{t}=2.0 \mathrm{~s}$ by tripping the corresponding line. Two types of short-circuit faults are used for test. They are single line to ground and line-to-line faults. The results are compared with that from the traditional method. Tables II and III show the results of the two faults respectively. In Table II, generator rotor angles (in degrees) at time $\mathrm{t}=1.5 \mathrm{~s}$ and $t=2.0 \mathrm{~s}$ are listed for comparison. It can be seen the rotor angle from new method is exactly the same as that from the conventional (old) method. This makes sense because of the strict derivation of the new method. In Table III, similar comparison is made to the L-L fault. The results are satisfactory as well.

Another test case is for accuracy comparison when network element parameter asymmetry is considered or ignored. The fault used is the line-to-line fault mentioned above. Lines 17-18 and 37-38 are selected to demo the asymmetric parameters of elements. The unbalance is simulated by setting the corresponding nondiagonal elements in (10) to be $10 \%$ and $20 \%$ of the relevant diagonal elements. The simulation results are shown in Table IV. Table IV shows that ignoring the unbalance of elements may lead to some errors, but the error is pretty small and has no noticeable influence to the stability results. This is complied with the industrial experience that the network unbalances can be ignored in transient stability analysis if the element parameter unbalance is not severe.

\section{B. Results From Test System 2}

The IEEE 20-machine, 118-bus system is adopted to test the effectiveness and efficiency of the new method in large power systems. The selected faults are applied at the from-bus of line 15-31 and the fault types are still $1 \mathrm{~L}-\mathrm{G}$ and L-L faults. Tables V and VI show the generator rotor angles (in degrees) at time $\mathrm{t}=$ $1.5 \mathrm{~s}$ and $\mathrm{t}=2.0 \mathrm{~s}$ of each fault respectively. The results show the new method is effective and with good accuracy for largescale power system transient stability simulation. 
TABLE II

COMPARISON OF GENERATOR ANGLES UNDER 1L-G FAULT

\begin{tabular}{ccccc}
\hline Gen. No. & \multicolumn{2}{c}{$\mathrm{t}=1.5 \mathrm{~s}$} & $\mathrm{t}=2.0 \mathrm{~s}$ \\
& \multicolumn{1}{c}{ New } & Old & New & Old \\
\cline { 2 - 5 } & -0.01952 & -0.01952 & 3.81217 & 3.81217 \\
1 & 19.83951 & 19.83951 & 8.95631 & 8.95631 \\
2 & 25.19556 & 25.19556 & 16.02137 & 16.02137 \\
3 & 0.15171 & 0.15171 & -1.88792 & -1.88792 \\
4 & -2.77908 & -2.77908 & -11.23738 & -11.23738 \\
5 & -6.56226 & -6.56226 & -16.65568 & -16.65568 \\
6 & & & & \\
\hline
\end{tabular}

TABLE III

COMPARISON OF THE GENERATOR ANGLES UNDER L-L FAULT

\begin{tabular}{ccccr}
\hline Gen. No. & \multicolumn{2}{c}{$1.5 \mathrm{~s}$} & \multicolumn{2}{c}{$2.0 \mathrm{~s}$} \\
\cline { 2 - 5 } & New & Old & New & -409.68955 \\
\hline 1 & -238.96178 & -238.96178 & -409.68955 & 1033.77562 \\
2 & 507.30738 & 507.30738 & 1033.77562 & 1040.13302 \\
3 & 515.00479 & 515.00479 & 1040.13302 & 639.42851 \\
4 & 399.54587 & 399.54587 & 639.42851 & 666.74384 \\
5 & 387.49828 & 387.49828 & 666.74384 & 635.83202 \\
6 & 401.46288 & 401.46288 & 635.83202 & \\
\hline
\end{tabular}

TABLE IV

COMPARISON OF GENERATOR ANGLES AT $\mathrm{t}=3.0 \mathrm{~s}$ WITH ASYMMETRY OF ELEMENTS CONSIDERED OR IGNORED

\begin{tabular}{cccc}
\hline Gen. No. & $\begin{array}{c}\text { Unbalance } \\
\text { ignored }\end{array}$ & $\begin{array}{c}10 \% \text { Unbalances } \\
\text { considered }\end{array}$ & $\begin{array}{c}20 \% \text { Unbalances } \\
\text { considered }\end{array}$ \\
\hline 1 & -784.17318 & -783.25000 & -773.74501 \\
2 & 1808.93094 & 1811.55382 & 1845.25903 \\
3 & 1828.91526 & 1831.15955 & 1863.43548 \\
4 & 1413.55858 & 1413.46498 & 1392.19314 \\
5 & 1415.76599 & 1415.98106 & 1398.61642 \\
6 & 1410.32284 & 1410.32004 & 1389.36931 \\
\hline
\end{tabular}

TABLE $\mathrm{V}$

GENERATOR ANGLES UNDER 1L-G FAULT

\begin{tabular}{ccccc}
\hline Gen. No & \multicolumn{2}{c}{$1.5 \mathrm{~s}$} & \multicolumn{2}{c}{$2.0 \mathrm{~s}$} \\
\cline { 2 - 5 } & New & Old & New & Old \\
\hline 1 & 6.022870 & 6.022870 & 5.91497 & 5.91497 \\
2 & 17.60815 & 17.60815 & 18.74295 & 18.74295 \\
3 & -21.11169 & -21.11169 & -17.81099 & -17.81099 \\
4 & -1.871860 & -1.871860 & 1.88291 & 1.88291 \\
5 & 0.591950 & 0.591950 & 4.10134 & 4.10134 \\
6 & -22.86725 & -22.86725 & -18.73223 & -18.73223 \\
\hline
\end{tabular}

TABLE VI

GENERATOR ANGLES UNDER L-L FAULT

\begin{tabular}{ccccc}
\hline Gen. No. & \multicolumn{2}{c}{$\mathrm{t}=1.5 \mathrm{~s}$} & $\mathrm{t}=2.0 \mathrm{~s}$ & Old \\
\cline { 2 - 5 } & New & Old & 3.04657 & 3.04657 \\
\hline 1 & 7.835330 & 7.835330 & 14.39098 & 14.39098 \\
2 & 19.00874 & 19.00874 & -8.92535 & -8.92535 \\
3 & -35.58223 & -35.58223 & 24.67662 & 24.67662 \\
4 & -29.26003 & -29.26003 & 25.80453 & 25.80453 \\
5 & -28.65641 & -28.65641 & 4.15224 & 4.15224 \\
6 & -49.13272 & -49.13272 & & \\
\hline
\end{tabular}

The computer time for the proposed method and the conventional method is the same (no difference is found) for all the test cases, which means the new method is efficient in transient stability simulation. 


\section{CONCLUSION}

A unified approach has been proposed in the paper to processing unbalanced operating conditions in transient stability calculations. The suggested method combined advantages of both abc phase coordinates and 012 sequence coordinates. The former is used to describe faults since it is suitable to simultaneous faults and the latter is used for time simulation for its sparse nature. Novel fault description and coordinate conversion formulation is presented. With the new method, the sequence network constitution can be avoided; multiple and simultaneous faults and element parameter unbalance can be simulated easily; and the sparse technology can still be applied for study efficiency. Computer test results show clearly the effectiveness and efficiency of the new method.

\section{REFERENCES}

[1] B. M. Weedy and B. J. Cory, Electric Power Systems. Chichester, U.K.: Wiley, 1998.

[2] Z. X. Han, "Generalized method of analysis of simultaneous faults in electric power system," IEEE Trans. Power App. Syst., vol. PAS-102, pp. 3933-3942, 1982.

[3] N. A. Ong et al., "Power system analysis of polyphase network in direct phase quantities," in Proc. 8th Mediterranean Electrotechnical Conf. (Melecon), Bari, Italy, 1996, pp. 1642-1645.

[4] L. Roy and N. D. Rao, "Exact calculation of simultaneous faults involving open-conductors and line-to-ground short circuits on inherently unbalanced power system," IEEE Trans. Power App. Syst., vol. 101, no. 8, pp. 2738-2746, Aug. 1982.

[5] Y. Sekine, Transient State Analysis of Power System, China: Mechanical Industry Press, 1989.

[6] V. Brandwajn and W. F. Tinney, "Generalized method for fault analysis," IEEE Trans. Power App. Syst., vol. PAS-104, no. 6, pp. 1301-1306, 1985.

[7] V. C. Strezoski and D. D. Bekut, "A canonicai model for the study of faults in power systems," IEEE Trans. Power Syst., vol. 6, no. 4, pp. 1493-1499, Nov. 1991.

[8] B. M. Zhang and J. Yang, "A cannonical method of network manipulation for unsymmetrical changes in power system network," in Proc. ICPST, Beijing, China, 1994, pp. 131-136.

[9] Z. Guo and Z. Liu, "Fast transient stability simulation by higher order taylor series expansions," Proc. CSEE, vol. 11, no. 3, pp. 8-16, 1991.

[10] P. Kundur, Power System Stability and Control. Englewood Cliffs, NJ: McGraw-Hill, 1994.
[11] M. S. Chen and W. E. Dillon, "Power system modeling," Proc. IEEE, vol. 62, no. 7, pp. 901-915, Jul. 1974.

[12] D. Z. Xia, "On-line transient evaluation by system decomposition aggregation and high order derivatives," IEEE Trans. Power App. Syst., vol. PAS-102, no. 7, pp. 2038-2046, 1983.

Xuefeng Bai received the B.Eng., M.Eng., and Ph.D. degrees from the Harbin Institute of Technology, Harbin, China, in 1996, 1998, and 2002, respectively.

His research interests are power system transient stability analysis and dynamic security assessment.

Tong Jiang received the B.Eng., M.Eng., and Ph.D. degrees from the Harbin Institute of Technology, Harbin, China, in 1992, 1995, and 2002, respectively.

His research interests are fault analysis and polyphase transmission analysis.

Zhizhong Guo received the B.Eng., M.Eng., and Ph.D. degrees from the Harbin Institute of Technology (HIT), Harbin, China.

$\mathrm{He}$ is currently a Principal Professor at the Electric Power System Research Center at HIT. His research interests are power system stability analysis and control and the application of optic measurement techniques in power systems.

Zheng Yan received the B.Sc. degree from Shanghai Jiaotong University, Shanghai, China, in 1984, and the M.Sc. and Ph.D. degrees from Tsinghua University, Beijing, China, in 1987 and 1991, respectively, all in electrical engineering.

He joined the University of Hong Kong as an Industry Fellow in 2001. His research interests are in dynamic security analysis and optimal power flow.

Yixin Ni (SM'94) received the B.Eng., M.Eng., and Dr.Eng. degrees in electrical engineering from Tsinghua University, Beijing, China.

She was a former Professor and Director of the National Power System Lab, Tsinghua University. She is currently with the University of Hong Kong. Her interests are power system stability and control, FACTS, AI applications in power systems, and power markets. 